Nace Pušnik

Stanislav Praček

DOI: $10.21278 /$ TOF.40303

ISSN 1333-1124

eISSN 1849-1391

\title{
THE EFFECT OF WINDING ANGLE ON UNWINDING YARN
}

\begin{abstract}
Summary
In the production of fabric, the unwinding of thread occurs in the warping and weft insertion processes. In order to achieve low and constant tension of thread or yarn it is necessary to optimize the process of unwinding. Computer simulations are now in use for this purpose, so it is important to obtain a mathematical description of yarn motion. This article is devoted to the derivation of boundary conditions that considerably affect the form of the balloon. In this way, a mathematically well defined model of yarn unwinding will be obtained which could be solved by using the tools of numerical mathematics. The unwinding of yarn from an optimally designed package can be simulated and this knowledge can be used to find an optimal design of packages.
\end{abstract}

Keywords: balloon theory, winding angle, yarn unwinding, oscillations in yarn, unwinding simulations.

\section{Introduction}

Many textile processes include yarn unwinding as an essential step. Oscillations which are included in yarn should be low and constant. Unwinding processes are important when producing high quality garments. All this depends on the characteristics of the optimized process.

D. Padfield is considered to be a pioneer of the unwinding and balloon formation theory. Because of her, Mack's equation was modified and now it includes the theory on the Coriolis force [1,2]. Calculation of multiple balloons and balloons formed during unwinding from packages with different geometry (for example conic packages) is also based on her theory [3]. Similar equations including the effect of gravity and tangential air drag were made by Kothari and Leaf $[4,5]$. They used elaborate numerical calculations to show small contribution of two forces to yarn dynamics. The mathematical theory of perturbations to correctly eliminate the time dependence of motion in stationary conditions was formulated by Fraser et. al. [6,7]. They showed that the entire time dependence can be shifted to moving boundary conditions. For this reason, the problem of partial differential equations (initial value problem) can be reduced to a boundary problem. Solving these equations is somehow easier. In our case and based on these foundations we further simplified the problem. Our solution will show a simple model function describing the package. We will show how the winding angle and the apex angle influence the dimensionless angular velocity of the yarn during the unwinding. This velocity has a large influence on the tension that we wish to reduce [8-11]. 


\section{The equation of motion of yarn}

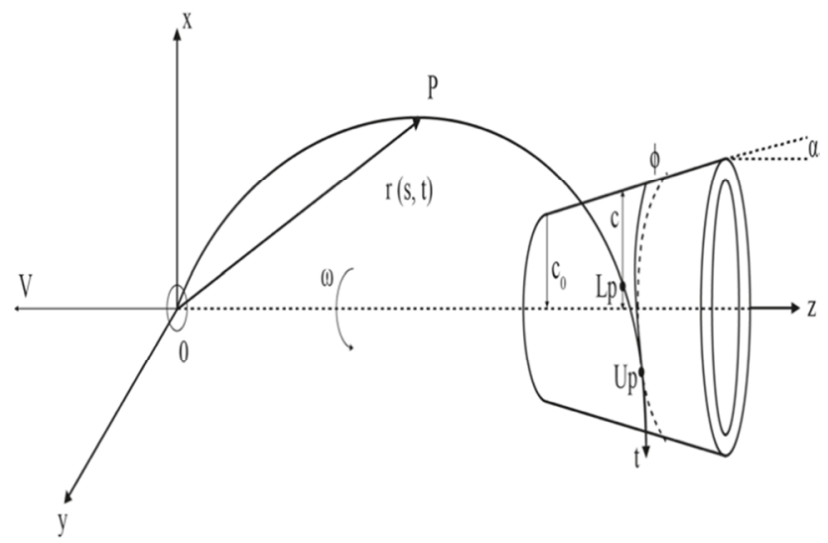

Fig. 1 Yarn unwinding from a cylindrical package.

The yarn is unwinding at a speed $\mathrm{V}$ through the guide $\mathrm{O}$ that is also the origin of the coordinate system, Fig.1. The yarn is rotating around the z-axis at an angular velocity $\omega$. At the lift-off point Lp the yarn lifts from the package and forms a balloon. At the unwinding point Up the yarn starts to slide on the surface of the package. Angle $\phi$ is the winding angle of the yarn on the package. Its magnitude is the angle between the yarn at the unwinding point and the tangential direction at the package surface, while its sign determines whether the yarn is being withdrawn in the direction from the front end toward the rear end of the package $(\phi>0)$ or from the rear end toward the front end $(\phi<0)$ [6-9].

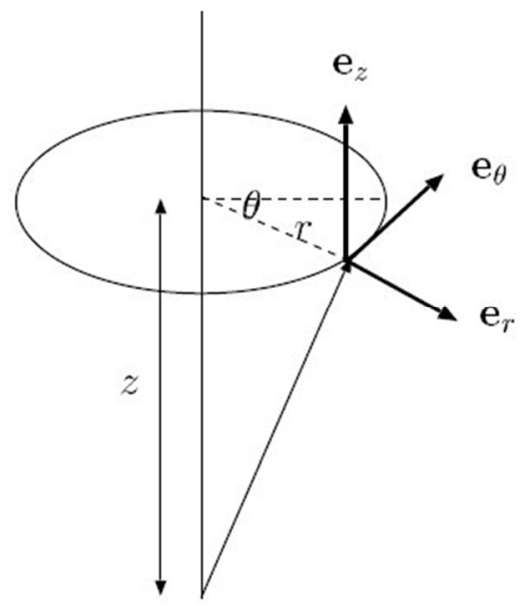

Fig. 2 The cylindrical coordinate system.

The yarn is parametrised with arc length $\mathrm{s}$ ( $\mathrm{s}$ is therefore the length of yarn from the origin of the coordinate system to the given point on the yarn). The coordinates of a point are given by $\mathrm{r}$, the radial distance from the axial axis, $\theta$, the polar angle and $\mathrm{z}$, the vertical distance from the origin, Fig.2. It should be kept in mind that each point has its own triplet of the base vectors $\mathrm{e}_{\mathrm{z}}, \mathrm{e}_{\theta}, \mathrm{e}_{\mathrm{r}}$, pointing in the vertical, tangential and radial direction, respectively. The radius vector $\mathbf{r}(s, t)$ pointing to a point on the yarn can be decomposed along the radial and vertical directions: $\mathbf{r}(s, t)=\mathrm{r}(s, t) \mathbf{e}_{r}(\theta(s, t), t)+\mathrm{z}(s, t) \mathbf{e}_{\mathrm{z}}(t)$. We have emphasized that coordinates of the point depend explicitly on both the time of observation $t$ and on the arc length $s$, where the point is located at given time $\mathbf{t}$. The vector $\mathbf{t}(s, t)$ is the vector tangential to the yarn at the given point; it equals the partial derivative of the radius vector $\mathbf{r}$ with respect to the arc-length $\mathrm{s}$, that is $\mathbf{t}=\partial \mathbf{r} / \partial \mathrm{s}$. 
The equation of motion of the yarn was derived in the previous paper $[6,12]$ :

$$
\rho\left(D^{2} \mathbf{r}+2 \boldsymbol{\omega} \times D \mathbf{r}+\boldsymbol{\omega} \times(\boldsymbol{\omega} \times \mathbf{r})+\dot{\omega} \times \mathbf{r}\right)=\frac{\partial}{\partial s}\left(T \frac{\partial \mathbf{r}}{\partial s}\right)+\mathbf{f}
$$

The vector $\mathbf{f}$ denotes the linear density of the external forces which are applied to the segment of yarn under discussion. In the part of the yarn which forms the balloon, $\mathbf{f}$ is the air drag force;

$$
\mathbf{f}=-D_{\mathrm{n}}\left|\mathbf{v}_{\mathbf{n}}\right| \mathbf{v}_{\mathrm{n}}
$$

where $D_{n}$ is the air drag coefficient and $\mathbf{v}_{\mathbf{n}}$ is the normal component of the velocity. When, however, the yarn is sliding on the package surface, the quantity $\mathbf{f}$ is related to the friction between the yarn and the package surface:

$$
\mathbf{f}=-\mu \mathrm{nv} /|\mathbf{v}|+\mathbf{n e}_{\mathrm{r}}
$$

Here $\mathrm{n}$ is the linear density of the normal component of the force between the yarn and the surface, $\mathbf{e}_{\mathrm{r}}$ is the unit vector in the radial direction, $\mathbf{v} /|\mathbf{v}|$ is the unit vector in the direction of the yarn. When the yarn slides on the surface, it thus experiences the normal force ne $\mathbf{e}_{\mathrm{r}}$ and the friction force $-\mu \mathrm{nv} /|\mathbf{v}|$. The operator $\mathrm{D}$ is the differential operator of the total time derivative following the motion of a point on the yarn. The unwinding speed, i.e., the speed with which the yarn is being withdrawn through the eyelet, is denoted by $\mathrm{V}$.

\subsection{Introduction of dimensionless quantities}

Before we continue the discussion of the equations of the yarn we introduce dimensionless quantities. In this way, we minimize the number of three parameters and simplify the analysis of the equations. Moreover, the dimensionless quantities are more convenient for numerical computations.

We will write a bar over the symbol of quantity to denote that it is a dimensionless quantity.

The length will be expressed in multiples of the radius of a package $c$. We get

$$
\overline{\mathbf{r}}=\mathbf{r}=/ c, \quad \bar{r}=r / c, \quad \bar{z}=z / c, \quad \bar{s}=s / c .
$$

Time will be expressed in multiples of the period of rotation (of the package around its axis $z$ ) $\tau_{0}=2 \pi / \omega$. It is even better if we omit the factor $2 \pi$ and express the time in multiples of $\tau=1 / \omega$ :

$$
\bar{t}=t / \tau=\omega t
$$

Let $V$ be the speed at which we pull the yarn. We will express the speed $\mathbf{v}$ and the speed $\mathbf{v}_{\mathrm{n}}$ as multiples of $V$.

$$
\overline{\mathbf{v}}=\mathbf{v} / V, \overline{\mathbf{v}}_{n}=\mathbf{v}_{n} / V
$$

We also introduce the force linear densities $\mathbf{f}$ and $\mathbf{n}$ and with the tension $T$. Tension is expressed in the same units as force (i.e. $\mathrm{N}=\mathrm{kgm} / \mathrm{s}^{2}$ ) while force linear densities are expressed in units $\mathrm{N} / \mathrm{m}$.

We can now introduce dimensionless quantities

$$
\overline{\mathbf{f}}=\frac{\mathbf{f} c}{\rho V^{2}}, \overline{\mathbf{n}}=\frac{\mathbf{n} c}{\rho V^{2}}, \bar{T}=\frac{T}{\rho V^{2}}
$$


We also introduce the dimensionless differential operator $\bar{D}$ as

$$
\bar{D}=\Omega \frac{\partial}{\partial \bar{t}}-\frac{\partial}{\partial \bar{s}}
$$

where

$$
\Omega=\frac{c \omega}{V}
$$

the parameter $\Omega$ is the dimensionless angular velocity, which will be often used in the sequel. The relation between the operator $D$ and $\bar{D}$ is $D=\frac{V}{c} \bar{D}$

When we rewrite the equation of motion with these dimensionless quantities we obtain

$$
\bar{D}^{2} \overline{\mathbf{r}}+2 \Omega \times \bar{D} \overline{\mathbf{r}}+\Omega \times(\Omega \times \overline{\mathbf{r}})+\Omega \frac{\partial \Omega}{\partial \bar{t}} \times \overline{\mathbf{r}}=\frac{\partial}{\partial \bar{s}}\left(\bar{T} \frac{\partial \overline{\mathbf{r}}}{\partial \bar{s}}\right)+\overline{\mathbf{f}}
$$

Let us now express the air resistance force and the friction force with dimensionless quantities.

For the air resistance force we get

$$
\begin{aligned}
\overline{\mathbf{f}} & =\mathbf{f} \frac{c}{\rho V^{2}} \\
& =-D_{n}\left|\mathbf{v}_{n}\right| \mathbf{v}_{n} \frac{c}{\rho V^{2}} \\
& =-D_{n}\left|\overline{\mathbf{v}}_{n}\right| \overline{\mathbf{v}}_{n} \frac{c}{\rho} \\
& =-\frac{p_{0}}{16}\left|\overline{\mathbf{v}}_{n}\right| \overline{\mathbf{v}}_{n},
\end{aligned}
$$

Where we introduced the following coefficient of air resistance

$$
p_{0}=\frac{16 c D_{n}}{\rho} .
$$

The number 16 is here for historical reasons, see [3]. This definition is now standard and we need to compare our results with other results.

We also need expressions for $\overline{\mathbf{v}}$ and $\overline{\mathbf{v}}_{n}$. We get

$$
\begin{aligned}
\overline{\mathbf{v}} & =\mathbf{v} / V \\
& =\frac{1}{V}(D r+\omega \times r) \\
& =\frac{1}{V}\left(\frac{V}{c} \bar{D} c \overline{\mathbf{r}}+\omega \times c \overline{\mathbf{r}}\right) \\
& =\bar{D} \overline{\mathbf{r}}+\frac{c}{V} \omega \times \overline{\mathbf{r}} \\
& =\bar{D} \overline{\mathbf{r}}+\Omega \times \overline{\mathbf{r}},
\end{aligned}
$$

for the first one and 


$$
\begin{aligned}
\overline{\mathbf{v}}_{n} & =\frac{1}{V} \mathbf{v}_{n} \\
& =\frac{1}{V} \frac{\partial \mathbf{r}}{\partial s} \times\left(\mathbf{v} \times \frac{\partial \mathbf{r}}{\partial s}\right) \\
& =\frac{\partial \overline{\mathbf{r}}}{\partial \bar{s}} \times\left(\overline{\mathbf{v}} \times \frac{\partial \overline{\mathbf{r}}}{\partial \bar{s}}\right) .
\end{aligned}
$$

for the second one.

The expression for the dimensionless force linear density of the package of yarn is similar to the usual (dimensional) expression [5].

$$
\overline{\mathbf{f}}=-\mu \bar{n} \frac{\mathbf{v}}{|\mathbf{v}|}+\bar{n} \mathbf{e}_{r}
$$

It is clear from the form of the dimensionless equations that the minimal number of parameters completely characterizing the solution is equal to 3 . The parameters are:

- $\Omega$, the dimensionless angular velocity rotating yarn around its axis;

- $\quad p_{0}$, the coefficient of air resistance;

- $\quad \mu$, the coefficient of friction.

\section{Relation between the angular velocity and the winding angle of packages}

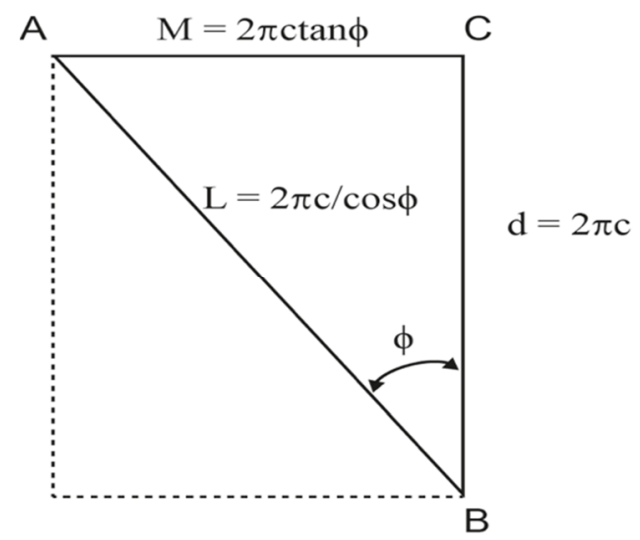

Fig. 3 Cross-section of a package along a line parallel to the package axis.

We will derive the relation between the angular velocity of the yarn during unwinding $\omega$, the unwinding speed $V$, and the package radius $\mathrm{c}$ at the lift-off point Lp (Fig.1). The derivation is applicable in the quasi-stationary approximation which consists of neglecting the variation of the system parameters (in particular of the winding angle $\phi$ ) during a single period of the balloon motion around the z-axis. The winding angle is positive if during the unwinding the lift-off point moves in the direction of higher values of the coordinate $\mathrm{z}$ (this corresponds to the unwinding in the backward direction). The winding angle is negative if during the unwinding the lift-off point moves in the direction of lower values of $z$ (the unwinding in the forward direction) [6-9]. We introduce a new auxiliary quantity $V_{1}$, the velocity of the yarn at the lift-off point. This velocity is not necessarily equal to the unwinding velocity $V$. Instead we have [2,3]:

$$
V_{1}=V+\frac{\mathrm{d} s}{\mathrm{~d} t}
$$


where $\mathrm{s}$ is the length of the yarn forming the balloon (i.e., between the lift-off point and the eyelet). $V_{1}$ is composed of the unwinding velocity and the time derivative of the length $\mathrm{s}$ : this is the "velocity" of the extension of the yarn in the balloon. This corresponds to the fact that in a given time interval, some of the yarn unwinds through the eyelet, while some yarn remains in the balloon and makes it longer. Since by definition $V_{1}=L / t$ we obtain

$$
V_{1}=\frac{c \omega}{\cos \phi}
$$

The quantity $\mathrm{d} s / \mathrm{d} t$ can be computed by the chain rule

$$
\frac{\mathrm{d} s}{\mathrm{~d} t}=\frac{\mathrm{d} s}{\mathrm{~d} z_{1}} \frac{\mathrm{d} z_{1}}{\mathrm{~d} t}
$$

where $\mathrm{z}_{1}$ is the coordinate $\mathrm{z}$ of the lift-off point, which moves up and down the package during the unwinding.

$$
\frac{\mathrm{d} z_{1}}{\mathrm{~d} t}=\frac{M}{t}=\frac{2 \pi c \tan \phi}{\frac{2 \pi}{\omega}}=c \omega \tan \phi,
$$

Let us consider the cylinder unfolded into a plane by cutting the surface along a line parallel to the cylindrical axis, Fig. 3. During a single period $t=2 \pi / \omega$ the balloon revolves once around the axis. During the same time the lift-off point likewise makes a single roundtrip around the package: it moves from point $A$ to point $B$ on the unfolded cylinder surface. If the lift-off point performed more or less than exactly one full rotation around the package, this would be in contradiction with our assumption of quasi-stationary conditions. The length of the yarn which is being unwound during a single period is therefore equal to $L=A B=$ $2 \pi \mathrm{c} / \cos \phi$. The position of the lift-off point on the unfolded cylinder is then $B$, which is equivalent to the point $C$ (points $B$ and $C$ are actually the same point if we take the periodic boundary conditions in the tangential direction of the cylinder surface into account). Consequently, the lift-off point moves for $M=\mathrm{AC}=2 \pi \operatorname{ctan} \phi$ along the package surface. In the quasi-stationary approximation we can then interpret this change in the following way: the arc-length of the yarn forming the balloon has increased by $M$, while the yarn segment with the length $L-M$ has been unwound through the eyelet. The yarn velocity is thus $V=(L-M) / t$. The angular velocity that we are interested in is therefore

$$
\omega=\frac{2 \pi}{t}=\frac{2 \pi V}{L-M}=\frac{V}{\frac{c}{\cos \phi}-c \tan \phi}=\frac{V \cos \phi}{c(1-\sin \phi)}
$$

The movements along the $\mathrm{z}$-axis are reduced by a factor of $\cos \alpha$ : as the lift-off point for $\mathrm{M}$, on the package surface, its coordinate $\mathrm{z}$ changes by $\mathrm{M} \cos \alpha$. It is easy to see that this is indeed the case if one considers a section of the package along the $\mathrm{z}$-axis. We therefore have

$$
\frac{\mathrm{d} z_{1}}{\mathrm{~d} t}=c \omega \tan \phi \cos \alpha
$$

When the unwinding is quasi-stationary, it holds approximately that the length of the balloon s changes by the amount equal to the displacement of the lift-off point along the z-axis. In mathematical terms this approximation is

$$
\frac{\mathrm{d} s}{\mathrm{~d} z_{1}}=1
$$


It is not possible to obtain a better approximation without performing a full numerical solution to the yarn unwinding, as described in previous publications [4-9].

We now insert expressions $(17,18,19$ and 20) in Eq. (16) to obtain

$$
\frac{c \omega}{\cos \phi}=V+c \omega \tan \phi \cos \alpha
$$

which can be expressed in the final form as

$$
\omega=\frac{V \cos \phi}{c(1-\cos \alpha \sin \phi)}
$$

The quantity $\mathrm{c}$ in this equation is the effective radius of the conic package at the current liftoff point. If we denote the smallest radius by $\mathrm{c}_{0}$ and the current position of the lift-off point along the $\mathrm{z}$-axis by $\mathrm{z}$, where $\mathrm{z}=0$ corresponds to the package edge closest to the eyelet, then one has

$$
c=c_{0}+z \tan \alpha
$$

\section{Motion of the lift-off point during unwinding}

During unwinding the lift-off point moves up and down the package. Let us consider now the displacement of the point in an infinitesimally short time $\mathrm{d} t$. If the motion is analyzed in the cylindrical coordinate system ( $\mathrm{r} \theta \mathrm{z}$ ), Fig. 2, where $\mathrm{r}$ is the distance of the point from the package axis, $\theta$ is the polar angle, and $\mathrm{z}$ the "height", then the infinitesimal change of the polar angle is $\mathrm{d} \theta=\omega \mathrm{d} t$. The current angular velocity $\omega$ is computed using Eq. (20) for cylindrical package or using Eq. (24) for conical package. The displacement of the lift-off point along the perimeter of the package within the time $\mathrm{d} t$ is equal to $\mathrm{d} l=\operatorname{cd} \theta$. On cylindrical packages $\mathrm{c}$ is the package radius which is constant, while on conical packages we need to use the current effective radius which is determined by Eq. (25). By definition the winding angle $\phi$ is determined as the angle between the tangential line on the package surface, perpendicular to the package axis, and the tangential line to the yarn at the lift-off point. In mathematical terms, $\tan \phi=\mathrm{d} z / \mathrm{d} l$. The infinitesimal displacement of the point along the $\mathrm{z}$-axis is thus equal to

$$
\mathrm{d} z=c \omega \tan \phi \mathrm{d} t
$$

In equation (26) we insert the value of the winding angle at the current position of the lift-off point. This value is approximately constant as the lift-off point moves up or down the package, but its sign changes abruptly at the package edges. If the bottom edge is given by $\mathrm{z}=0$ and the upper one by $\mathrm{z}=\mathrm{Z}$, then the angle $\phi$ as a function of the coordinate $\mathrm{z}$ is a function which is equal to zero at $z=0$ and at $z=Z$ (since it has to change sign at the edges), and approximately equal to $\phi_{0}$ for other values of $z$. These requirements are met, for example, by the trigonometric function, such as sine function. This function should be modified so that it will change only slightly when the point moves up or down the packages. We can achieve this by raising the sine to a low fractional power, say $1 / 40$ (we have to be careful about the signs, so we take absolute value of sine function and restore the sign using the signum function)

$$
f(t)=\operatorname{sign}(\sin t)|\sin t|^{\frac{1}{40}}
$$


In equation (27) the winding angle has to be inserted in the layer which is currently being unwound, $\phi_{0}$. We get

$$
\phi(t)=\phi_{0} f(t)
$$

where $\phi_{0}$ is the maximal angle of winding. By this we mean the positive value of the winding angle, i.c., the characteristics of a given package.

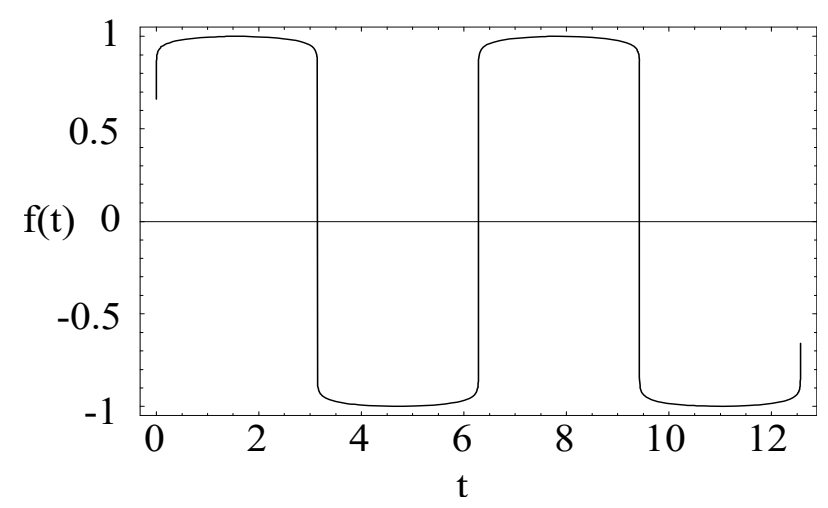

Fig. 4 Model function for winding angle.

Only one parameter remains in equation (10) without taking the external force term into account. It is the dimensionless angular velocity: $\Omega=\mathrm{c} \omega / V$. This is the single most important parameter in our model. There are two additional dimensionless parameters: $\mu$, the coefficient of friction between yarn and package, and the coefficient of air resistance $p_{0}$. These two parameters are approximately constant during unwinding, so they are less important. The coefficient of friction depends on the quality of the yarn and on the surface properties of the package. The quality of the surface is comparable for forward and backward unwinding direction, so that the coefficient of friction remains approximately constant.

By introducing the dimensionless angular velocity $\Omega$ (and using $\phi(t)=\phi_{0} f(t)$ ) we can rewrite equations (20) and (24) as:

$$
\Omega(\phi(t))=\frac{\cos (\phi(t))}{1-\sin (\phi(t))}
$$

and

$$
\Omega(\phi(t))=\frac{\cos (\phi(t))}{1-\cos (\alpha) \sin (\phi(t))}
$$

\section{Results of unwinding simulation}

We have performed simulations of unwinding from both cylindrical and conic packages. We showed the time dependence of the dimensionless angular velocity $\Omega=\Omega(\phi(t))$. As a side product of the calculation we also obtained the time dependence of the apex angle $\alpha$ and the time dependence of the winding angle $\phi(t)$. During unwinding in the forward direction this angle is negative, during unwinding in the backward direction it is positive. The time is expressed in units of the period (i.e., $2 \pi$ corresponds to one full period). 


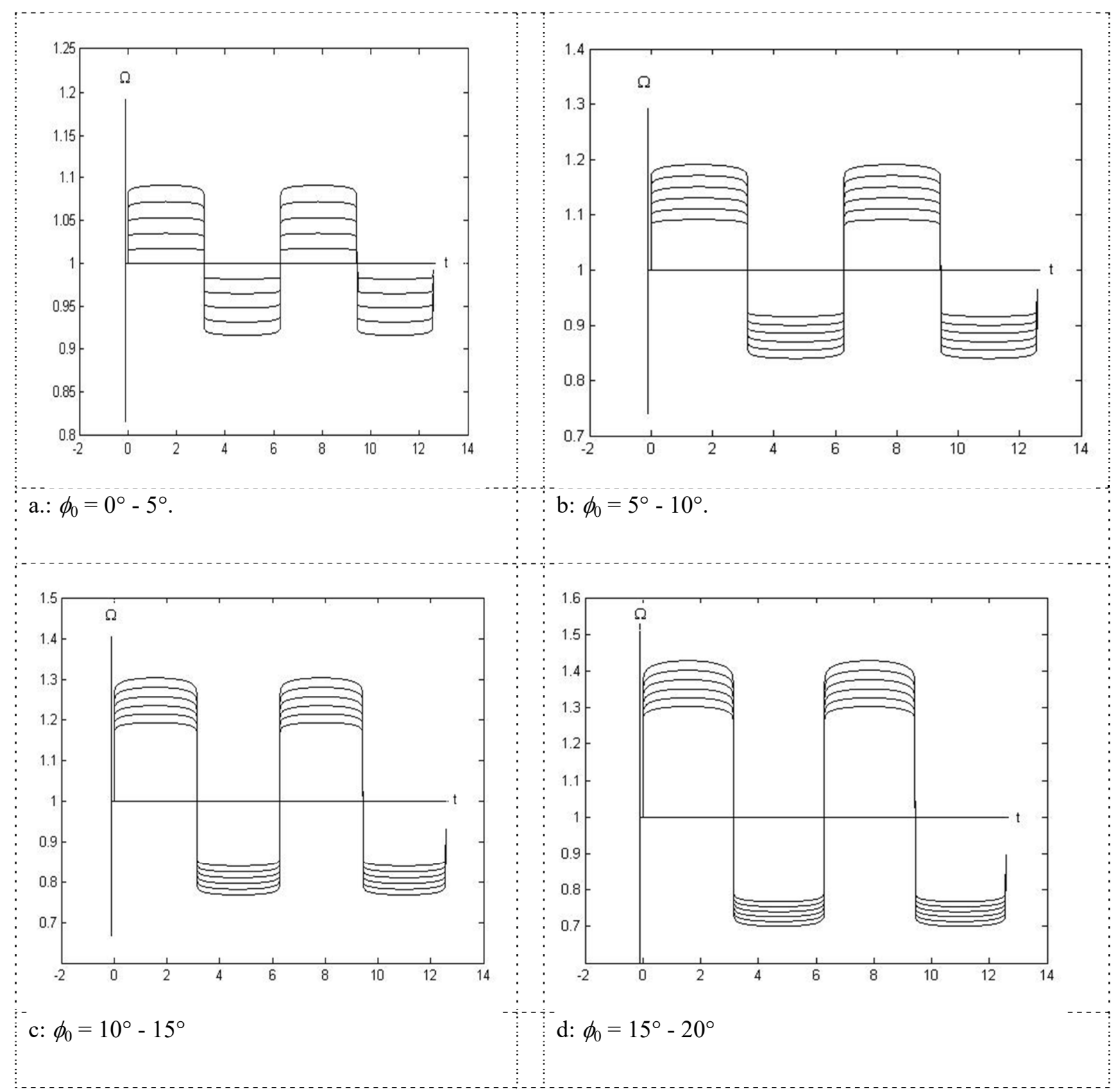

Fig. 5 Oscillations of dimensionless angular velocity $\Omega$ during unwinding of yarn from cylindrical packages, where $\phi_{0}=0^{\circ}-20^{\circ}$. 

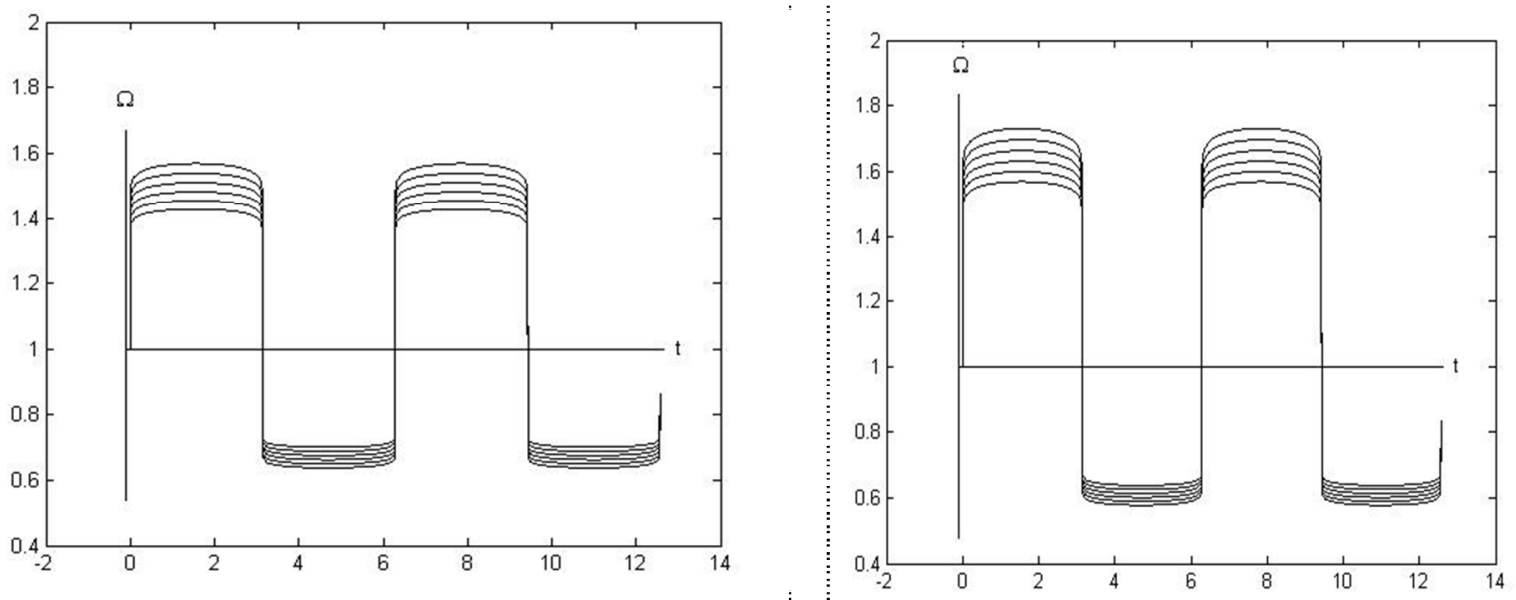

a.: $\phi_{0}=20^{\circ}-25^{\circ}$

b: $\phi_{0}=25^{\circ}-30^{\circ}$.
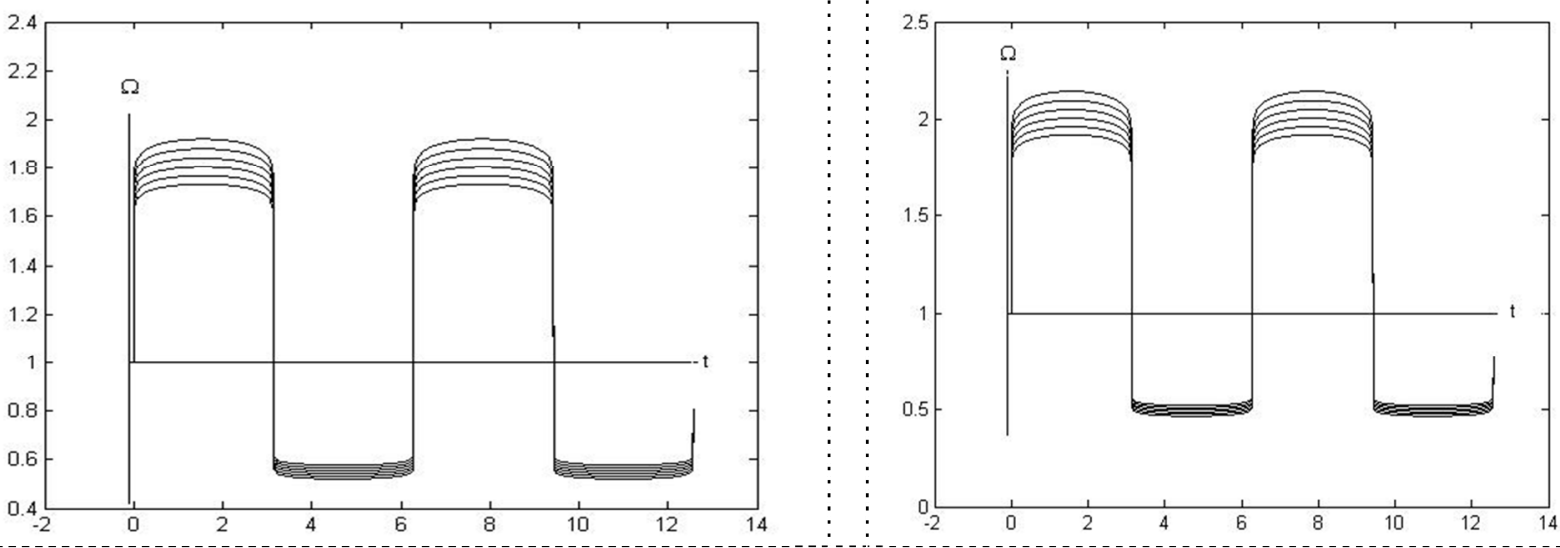

c: $\phi_{0}=30^{\circ}-35^{\circ}$

$\mathrm{d}: \phi_{0}=35^{\circ}-40^{\circ}$

Fig. 6 Oscillations of dimensionless angular velocity $\Omega$ during unwinding of yarn from cylindrical packages, where $\phi_{0}=20^{\circ}-40^{\circ}$. 
Table 1 Dimensionless angular velocity as function of winding angle $\phi$.

\begin{tabular}{|c|c|c|c|}
\hline Angle $\phi_{0}$ & $\Omega$ at winding backward & Angle $\phi_{0}$ & $\Omega$ at winding forward \\
\hline$\sim 0^{\circ}$ & 1 & $\sim 0^{\circ}$ & 1 \\
\hline $1^{\circ}$ & 1.0176 & $-1^{\circ}$ & 0.9827 \\
\hline $2^{\circ}$ & 1.0355 & $-2^{\circ}$ & 0.9657 \\
\hline $3^{\circ}$ & 1.0538 & $-3^{\circ}$ & 0.9490 \\
\hline $4^{\circ}$ & 1.0724 & $-4^{\circ}$ & 0.9325 \\
\hline $5^{\circ}$ & 1.0913 & $-5^{\circ}$ & 0.9163 \\
\hline $6^{\circ}$ & 1.1106 & $-6^{\circ}$ & 0.9004 \\
\hline $7^{\circ}$ & 1.1303 & $-7^{\circ}$ & 0.8847 \\
\hline $8^{\circ}$ & 1.1504 & $-8^{\circ}$ & 0.8693 \\
\hline $9^{\circ}$ & 1.1708 & $-9^{\circ}$ & 0.8541 \\
\hline $10^{\circ}$ & 1.1918 & $-10^{\circ}$ & 0.8391 \\
\hline $11^{\circ}$ & 1.2131 & $-11^{\circ}$ & 0.8243 \\
\hline $12^{\circ}$ & 1.2349 & $-12^{\circ}$ & 0.8098 \\
\hline $13^{\circ}$ & 1.2572 & $-13^{\circ}$ & 0.7954 \\
\hline $14^{\circ}$ & 1.2799 & $-14^{\circ}$ & 0.7813 \\
\hline $15^{\circ}$ & 1.3032 & $-15^{\circ}$ & 0.7673 \\
\hline $16^{\circ}$ & 1.3270 & $-16^{\circ}$ & 0.7536 \\
\hline $17^{\circ}$ & 1.3514 & $-17^{\circ}$ & 0.7400 \\
\hline $18^{\circ}$ & 1.3764 & $-18^{\circ}$ & 0.7265 \\
\hline $19^{\circ}$ & 1.4019 & $-19^{\circ}$ & 0.7133 \\
\hline $20^{\circ}$ & 1.4281 & $-20^{\circ}$ & 0.7002 \\
\hline $21^{\circ}$ & 1.4550 & $-21^{\circ}$ & 0.6873 \\
\hline $22^{\circ}$ & 1.4826 & $-22^{\circ}$ & 0.6745 \\
\hline $23^{\circ}$ & 1.5108 & $-23^{\circ}$ & 0.6619 \\
\hline $24^{\circ}$ & 1.5399 & $-24^{\circ}$ & 0.6494 \\
\hline $25^{\circ}$ & 1.5697 & $-25^{\circ}$ & 0.6371 \\
\hline $26^{\circ}$ & 1.6003 & $-26^{\circ}$ & 0.6249 \\
\hline $27^{\circ}$ & 1.6319 & $-27^{\circ}$ & 0.6128 \\
\hline $28^{\circ}$ & 1.6643 & $-28^{\circ}$ & 0.6009 \\
\hline $29^{\circ}$ & 1.6977 & $-29^{\circ}$ & 0.5890 \\
\hline $30^{\circ}$ & 1.7321 & $-30^{\circ}$ & 0.5774 \\
\hline $31^{\circ}$ & 1.7675 & $-31^{\circ}$ & 0.5658 \\
\hline $32^{\circ}$ & 1.8040 & $-32^{\circ}$ & 0.5543 \\
\hline $33^{\circ}$ & 1.8418 & $-33^{\circ}$ & 0.5430 \\
\hline $34^{\circ}$ & 1.8807 & $-34^{\circ}$ & 0.5317 \\
\hline $35^{\circ}$ & 1.9210 & $-35^{\circ}$ & 0.5206 \\
\hline $36^{\circ}$ & 1.9626 & $-36^{\circ}$ & 0.5095 \\
\hline $37^{\circ}$ & 2.0057 & $-37^{\circ}$ & 0.4986 \\
\hline $38^{\circ}$ & 2.0503 & $-38^{\circ}$ & 0.4877 \\
\hline $39^{\circ}$ & 2.0965 & $-39^{\circ}$ & 0.4770 \\
\hline $40^{\circ}$ & 2.1445 & $-40^{\circ}$ & 0.4663 \\
\hline
\end{tabular}



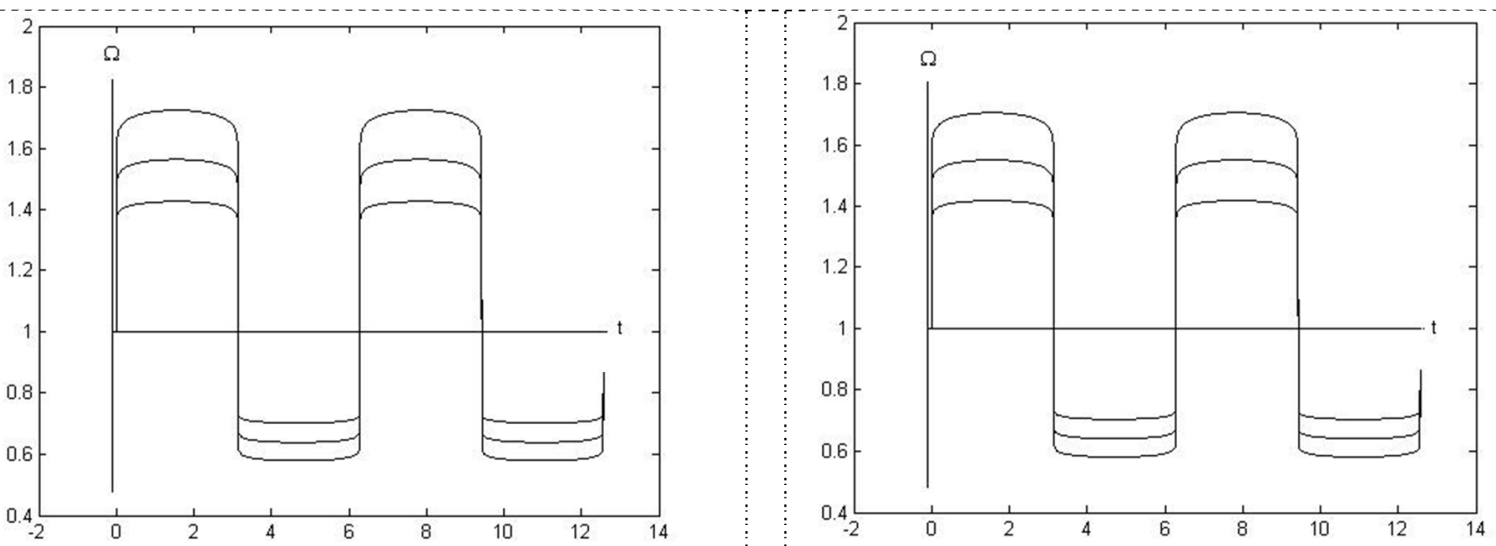

a.: $\phi_{0}=20^{\circ}-30^{\circ}, \alpha=5^{\circ}$.

b: $\phi_{0}=20^{\circ}-30^{\circ}, \alpha=10^{\circ}$.

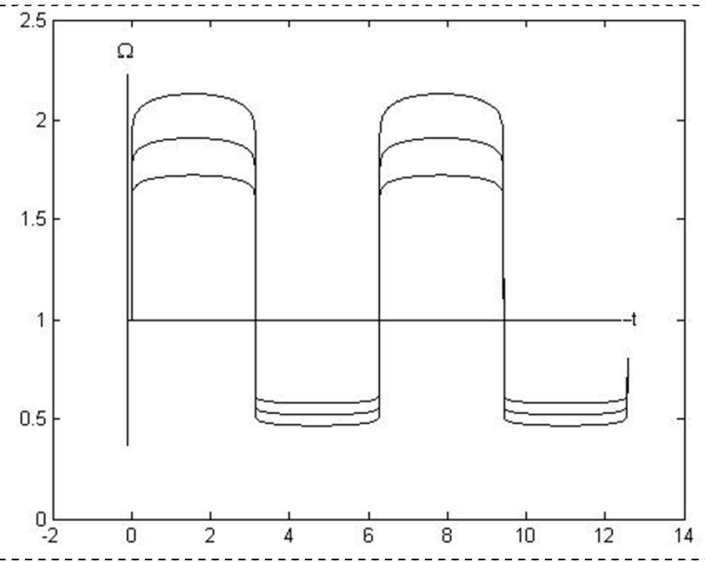

c: $\phi_{0}=30^{\circ}-40^{\circ}, \alpha=5^{\circ}$.

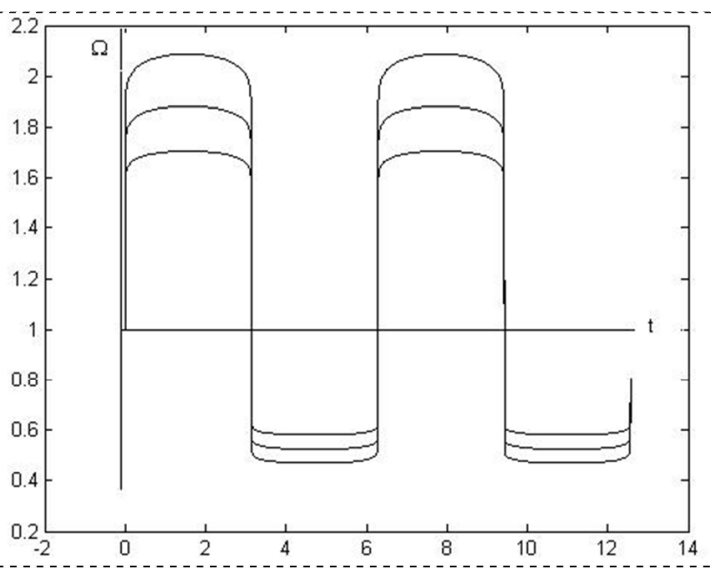

$\mathrm{d}: \phi_{0}=30^{\circ}-40^{\circ}, \alpha=10^{\circ}$.

Fig. 7 Oscillations of dimensionless angular velocity $\Omega$ during unwinding of yarn from conic packages, where $\phi_{0}=20^{\circ}-40^{\circ}$ and $\alpha=5^{\circ}$ to $10^{\circ}$.

Table 2 Dimensionless angular velocity as function of winding angle $\phi$ and apex angle $\alpha$.

\begin{tabular}{|c|c|c|c|}
\hline Apex angle $\alpha$ & Winding angle $\phi_{0}$ & $\Omega$ at winding backward & $\Omega$ at winding forward \\
\hline $0^{\circ}$ & $\sim 0^{\circ}$ & 1 & 1 \\
\hline $0^{\circ}$ & $5^{\circ}$ & 1.091 & 0.916 \\
\hline $0^{\circ}$ & $10^{\circ}$ & 1.1918 & 0.8391 \\
\hline $0^{\circ}$ & $15^{\circ}$ & 1.3032 & 0.7673 \\
\hline $0^{\circ}$ & $20^{\circ}$ & 1.4281 & 0.7002 \\
\hline $0^{\circ}$ & $25^{\circ}$ & 1.5697 & 0.6371 \\
\hline $0^{\circ}$ & $30^{\circ}$ & 1.7321 & 0.5774 \\
\hline $0^{\circ}$ & $35^{\circ}$ & 1.9210 & 0.5206 \\
\hline $0^{\circ}$ & $40^{\circ}$ & 2.1445 & 0.4663 \\
\hline $5^{\circ}$ & $\sim 0^{\circ}$ & 1 & 1 \\
\hline $5^{\circ}$ & $5^{\circ}$ & 1.0909 & 0.9166 \\
\hline $5^{\circ}$ & $10^{\circ}$ & 1.1908 & 0.8396 \\
\hline $5^{\circ}$ & $15^{\circ}$ & 1.3015 & 0.7679 \\
\hline $5^{\circ}$ & $20^{\circ}$ & 1.4253 & 0.7009 \\
\hline $5^{\circ}$ & $25^{\circ}$ & 1.5653 & 0.6378 \\
\hline $5^{\circ}$ & $30^{\circ}$ & 1.7255 & 0.5781 \\
\hline
\end{tabular}




\begin{tabular}{|c|c|c|c|}
\hline Apex angle $\alpha$ & Winding angle $\phi_{0}$ & $\Omega$ at winding backward & $\Omega$ at winding forward \\
\hline $5^{\circ}$ & $35^{\circ}$ & 1.9112 & 0.5213 \\
\hline $5^{\circ}$ & $40^{\circ}$ & 2.1299 & 0.4670 \\
\hline $10^{\circ}$ & $\sim 0^{\circ}$ & 1 & 1 \\
\hline $10^{\circ}$ & $5^{\circ}$ & 1.0897 & 0.9174 \\
\hline $10^{\circ}$ & $10^{\circ}$ & 1.1880 & 0.8410 \\
\hline $10^{\circ}$ & $15^{\circ}$ & 1.2963 & 0.7697 \\
\hline $10^{\circ}$ & $20^{\circ}$ & 1.4170 & 0.7029 \\
\hline $10^{\circ}$ & $25^{\circ}$ & 1.5524 & 0.6400 \\
\hline $10^{\circ}$ & $30^{\circ}$ & 1.7061 & 0.5803 \\
\hline $10^{\circ}$ & $35^{\circ}$ & 1.8825 & 0.5235 \\
\hline $10^{\circ}$ & $40^{\circ}$ & 2.0874 & 0.4691 \\
\hline
\end{tabular}

In equation (26) we presented a mathematical model to simulate the yarn unwinding from cylindrical packages and we showed the time dependence of the dimensionless angular velocity $\Omega$ from different winding angles $\phi_{0}$. We did the same for conical packages in equation (27) with the additional apex angle $\alpha$. If we want to know an exact solution of the mathematical model, we cannot find it in the pictures, because the values differ little one from another. For this reason, we inserted the values of $\Omega$ under different conditions in two tables. Table 1 introduces the yarn winding forward and backward from cylindrical packages; Table 2 contains the values of the yarn winding forward and backward from conical packages. It is clear that the effect of the typical apex angle on the dimensionless angular velocity is negligible for all practical purposes.

\section{Conclusions}

Speaking of the unwinding process, we are mostly interested in the maximal tensions in the yarn and the oscillations of the tension as a function of the unwinding speed. We aim to achieve the highest possible speed, while keeping the tension in the yarn and oscillations as low as possible. The variations of the dimensionless angular velocity (Figs. 5, 6 and 7) are more pronounced in packages with a high winding angle. It is therefore inadmissible to consider the winding angle as a small quantity and to neglect it in the first approximation. The lower the winding angle is the lower will the oscillations of the tension be. The maxima will then be lower and we will be able to increase the unwinding speed. The problem is that most of the winding machines in use throughout the world use the circumferential driving of the tube. This method of package winding makes it impossible to change the winding angle, while this is possible with winding machines using the direct driving of the tube. In recent times, the fraction of such winding machines in use is increasing.

\section{REFERENCES}

[1] Mack, C. (1953). Theoretical study of ring and cap spinning balloon curves (with and without air drag). J.Text.Inst 44 483-498. DOI: 10.1080/19447025308662612

[2] Padfielf,D.G (1956). A note on fluctuations of tension during unwinding. J.Text.Inst 47 301-308. DOI: 10.1080/19447027.1956.10750412

[3] Padfield, D. G. (1958). The Motion and Tension of an Unwinding Thread. Proc. R. Soc., vol. A245, 382407. DOI: $10.1098 /$ rspa.1958.0090 
[4] Kothari,V.K., Leaf G.A.V. (1979). The unwinding of yarns from packages, Part I: The theory of yarnunwinding. J.Text.Inst 70 (3)89-95. DOI: 10.1080/00405007908631523

[5] Kothari,V.K., Leaf G.A.V. (1979). The unwinding of yarns from packages, Part II: Unwinding from cilindrical packages. J.Text.Inst 70 (3)96-104. DOI: 10.1080/00405007908631524

[6] Fraser, W. B., Ghosh, T. K., Batra, S. K. (1992). On unwinding yarn from cylindrical package. Proc. R. Soc. Lond. A, 436 479-498. DOI: 10.1098/rspa.1992.0030

[7] Fraser, W. B., (1992). The effect of yarn elasticity on an unwinding balloon. J. Tex. Inst, 83 603-613. DOI: $10.1080 / 00405009208631235$

[8] Kong, X. M., Rahn, C. D., Goswami, B. C. (1999). Steady-state unwinding of yarn from cylindrical packages. Text. Res. J., 69, 4, 292-306. DOI: 10.1177/004051759906900409

[9] J.D.Clark, W.B.Fraser, D.M.Stump. (2001). Modelling of tension in yarn package unwinding. J.Engi.Mathema., 40, 59-75. DOI: 10.1023/A:1017525804392

[10] Ghosh, Batra and Murthy. (2001).Dynamic Analysis of Yarn Unwinding from Cylindrical Packages, Part I: Parametric Studies of the Two-Region problem. Text. Res. J., 71, 9, 771-778. DOI: $10.1177 / 004051750107100905$

[11] Ghosh, Batra and Murthy. (2001).Dynamic Analysis of Yarn Unwinding from Cylindrical Packages, Part II:The Three-region Analysis. Text. Res. J., 71, 10, 855-861.

[12] S. Pracek, (2007). Theory of string motion in the textile process of yarn unwinding. I.J. Nonlinear Science\&Numerical Simulation, 8, 3, 451-460. DOI: 10.1515/ijnsns.2007.8.3.451

[13] Roberson, J. A., Crowe, C. T. (1980) Engineering fluid dynamics. Houghton Mifflin Company, Boston, second edition.

[14] Giancoli, Douglas C. Physics for scientists \& engineers: with modern physics. 4th ed. Upper saddler River: Pearson Education International, cop. 2009

[15] Kim, Kun-Woo; Lee, Jae-Wook; Yoo, Wan-Suk (2012). Effect of gravity and tangential air resistance on unwinding cable. Nonlinear Dynamic, Vol.70, 1, 67-87. DOI: 10.1007/s11071-012-0431-1

Submitted: $\quad 03.8 .2015$

Accepted: $\quad 20.9 .2016$
Nace Pušnik

Stanislav Praček

University of Ljubljana, Faculty of Natural Sciences and Engineering, Aškerčeva. 12, SI-1000 Ljubljana, Slovenija 